\title{
Attitudes and Barriers towards Pre-Exposure Prophylaxis (Prep) among High-Risk HIV-Seronegative Men who have Sex with Men
}

Jill K Gersh ${ }^{1 *}$, Suzanne P Fiorillo ${ }^{2}$, Liam Burghardt ${ }^{2}$, Aran Cunningham Nichol ${ }^{2}$, Mark Thrun ${ }^{2,3}$ and Thomas B Campbell ${ }^{*}$

${ }^{1}$ Department of Medicine, University of Colorado School of Medicine, Colorado, USA

${ }^{2}$ Division of Infectious Diseases, Department of Medicine, University of Colorado School of Medicine, Colorado, USA

${ }^{3}$ Denver Public Health, Denver, Colorado, USA

\begin{abstract}
Objective: Recent studies have demonstrated the efficacy of antiretroviral preexposure prophylaxis (PrEP) for prevention of HIV-1 infection. The purpose of this study was to identify barriers to PrEP use among high HIV-risk men who have sex with men in Denver, Colorado.

Methods: A 19 question Likert-scale survey was used to assess knowledge of PrEP; attitudes towards cost, side effects, and alternative prevention methods; and the effects of PrEP on sexual behaviors and practices. The survey was administered at study entry and six months later to HIV seronegative, men who have sex with men and male-tofemale transgendered women who have sex with men and participate in high-risk sexual behavior.

Results: Between June and September 2013, 65 participants from the metropolitan Denver area completed the survey. Median age was 36 years (range; $20-52$ years); $88 \%$ were white, $6 \%$ Hispanic, and $1.5 \%$ African American; $65 \%$ had at least a college degree or higher and $27 \%$ had more than high school education. The reported number of sexual partners in the past six months ranged from zero to 150 partners (median 3 partners) and $75 \%$ reported condom use during all sexual encounters. Although $72.3 \%$ reported prior knowledge of PrEP only five participants $(7.7 \%)$ reported ever using PrEP in the past. Participants were most likely $(93.8 \%)$ to use PrEP in the future if they were in a monogamous relationship with an HIV-infected partner and least likely to use PrEP if it required out-of-pocket costs $(10.7 \%)$. Younger age was associated with decreased odds of future PrEP use even if PrEP was provided free of charge (OR $0.2,95 \% \mathrm{Cl} 0.1,1.0)$. Higher number of sexual partners in the preceding six months was associated with decreased odds of using condoms if taking PrEP in the future (aOR 0.2; $95 \% \mathrm{Cl} 0.1,0.8$ ). No changes in survey responses were noted between baseline and six months.
\end{abstract}

Conclusions: PrEP usage was uncommon among men at high risk for sexual acquisition of HIV infection and cost of antiretroviral drugs was a major barrier to future PrEP use. If PrEP is to have major impact on transmission of HIV-1, expanded efforts to decrease cost and increase community awareness of PrEP safety and efficacy are needed.

Keywords: Pre-exposure prophylaxis; PrEP; HIV; Vaccine; Prevention; HVTN 505; Barriers; Sexual behaviors

\section{Introduction}

Recent studies have demonstrated the protective effects of emtricitabine/tenofovir-DF as pre-exposure chemoprophylaxis (PrEP) for patients at high risk of HIV-1 infection [1,2]. The iPrex study demonstrated that co-formulated emtricitabine/tenofovir-DF reduced the incidence of HIV infection by $44 \%$ among seronegative male-tofemale transgendered women or men who have sex with men (MSM) [1]. More recently, the Partners PrEP Trial demonstrated protection from HIV transmission among serodiscordant heterosexual couples in Uganda and Kenya, with a $67 \%$ relative reduction of HIV incidence with tenofovir-DF alone and 75\% with emtricitabine/tenofovir-DF when compared to placebo [2]. Other trials have demonstrated similar findings; however efficacy in HIV infection rate reduction is highly dependent on adherence to the antiretroviral regimen [3,4]. Although emtricitabine/tenofovir-DF has a favorable safety profile, side effects such as nausea, renal toxicity, bone density loss, and liver toxicity can limit use [5].

Little information is available on the factors that limit PrEP use in community settings. Several small studies have demonstrated moderate interest in taking PrEP among at-risk populations, if it were available. There is modest correlation between risk behavior and preference for PrEP, and often little knowledge about PrEP [6-10]. Little is known about how other factors such as medication cost, issues affecting daily compliance, HIV education, and prevention counselling affect PrEP use. The present study evaluated attitudes and potential barriers to PrEP use among men who have sex with men or transgender women and are participants in a clinical trial of a candidate HIV vaccine. PrEP's high cost, side effect profile, and individual sexual behaviour were hypothesized to be the greatest stated barriers for PrEP use in this setting.

\section{Methods}

\section{Study population}

Participants were recruited from those taking part in the HVTN

*Corresponding authors: Jill K Gersh, Department of Medicine, University of Colorado School of Medicine, 12631 East 17th Avenue, Mail Stop B168 Aurora, CO 80045, USA, Tel: +1 509590 9052; Fax: +1 303724 4926; E-mail: jillgersh@gmail.com

Thomas Campbell, Division of Infectious Diseases, Department of Medicine University of Colorado School of Medicine, 12631 East 17th Avenue, Mail Stop B168 Aurora, CO 80045, USA, Tel: +1 509590 9052; Fax: +1 303724 4926; E-mail: thomas.campbell@ucdenver.edu

Received June 06, 2014; Accepted July 28, 2014; Published August 11, 2014

Citation: Gersh JK, Fiorillo SP, Burghardt L, Nichol AC, Thrun M, et al. (2014) Attitudes and Barriers towards Pre-Exposure Prophylaxis (Prep) among HighRisk HIV-Seronegative Men who have Sex with Men. J AIDS Clin Res 5: 335 doi:10.4172/2155-6113.1000335

Copyright: ( 2014 Gersh JK, et al. This is an open-access article distributed unde the terms of the Creative Commons Attribution License, which permits unrestricted use, distribution, and reproduction in any medium, provided the original author and source are credited. 
505 vaccine trial at the University of Colorado Hospital Clinical Research Site, Aurora, Colorado. Complete eligibility criteria for HVTN 505 participation have been published elsewhere [11]. Participants in HVTN 505 were HIV seronegative men who have sex with men and male-to-female transgendered women who have sex with men who had unprotected anal intercourse with one or more male or maleto-female transgender partners or anal intercourse with two or more male or male-to-female transgender partners in the past six months. HVTN 505 enrollment at the University of Colorado Hospital began in November 2010 and was halted in April 2013, after enrollment of 107 participants, following recommendations by an independent data and safety monitoring board (DSMB) which found that the candidate vaccine did not prevent HIV infection nor reduce viral load after HIV infection [11,12]. All HVTN 505 study participants at the University of Colorado Hospital site who remained HIV seronegative were eligible for the current study and were invited to complete a survey about attitudes and barriers toward PrEP usage.

\section{Recruitment}

Recruitment took place by telephone from June 2013 to September 2013. After an attempt to contact a participant was made, the date and outcome of the call was recorded. If no contact was made after three attempts, no further follow-up was undertaken. Once verbal informed consent was obtained using a standard script, a trained interviewer administered a brief survey by telephone and recorded participant responses on paper forms. Beginning January 2014, participants were contacted again to complete a six-month follow-up survey, using the same questionnaire. This study was approved by the Colorado Multiple Institutional Review Board (COMIRB) and followed the human experimentation guidelines of the US Department of Health and Human Services.

\section{Survey instrument}

The 19 question Likert-scale survey took appropriately 10-15 minutes to administer by telephone (Appendix 1). The survey included questions about demographics, awareness of PrEP, prior history of PrEP use, associated costs, perceived side effects, alternative methods of HIV prevention and individual sexual behavior and practices. Specific

\begin{tabular}{|c|c|c|}
\hline & Number (n) & Percent (\%) \\
\hline \multicolumn{3}{|l|}{ Race/Ethnicity } \\
\hline White/Non-Hispanic & 57 & 87.7 \\
\hline White/Hispanic & 4 & 6.2 \\
\hline Black & 1 & 1.5 \\
\hline Other & 3 & 4.6 \\
\hline \multicolumn{3}{|l|}{ Education } \\
\hline HS diploma or GED & 5 & 7.7 \\
\hline More than HS & 18 & 27.7 \\
\hline College graduate or higher & 42 & 64.6 \\
\hline Ever heard about PrEP & 47 & 72.3 \\
\hline Ever taken PrEP & 5 & 7.7 \\
\hline \multicolumn{3}{|l|}{ Sexual activity } \\
\hline Receptive "bottom" partner & 11 & 16.9 \\
\hline Insertive "top" partner & 11 & 16.9 \\
\hline Both & 39 & 60.0 \\
\hline \multirow[t]{2}{*}{ Neither } & 4 & 6.2 \\
\hline & Median & Range \\
\hline Age (in years) & 36 & $20-52$ \\
\hline No. partners in past 6 months: & 3 & $0-150$ \\
\hline Percentage $(\%)$ of condom use in past 6 months & 75 & $0-100$ \\
\hline
\end{tabular}

Table 1: Demographics of participants who completed the initial survey. questions were adapted from prior studies of PrEP awareness [5,13,14]. Participants verbally rated their likelihood of taking PrEP under 14 specific circumstances or conditions using a five-point Likert scale rating ("Definitely" (1), "very likely" (2), "somewhat likely" (3), "not very likely" (4), or "definitely not" (5)) to identify barriers to PrEP use. Data were collected without personal identifiable information.

\section{Data analysis}

Survey data were collected and managed using REDCap electronic data capture tools hosted at the University of Colorado Denver [15]. All analysis was performed using SAS, version 9.4 (SAS Institute, Cary, NC, USA). Demographic information, including age, race/ ethnicity, education, and sexual practices was collected and analyzed descriptively. Likert scale questions were grouped by Likert score and frequency of answers. For each survey question, likely responses were calculated as the combination of "definitely" (1), "very likely" (2), and "somewhat likely" (3), while unlikely responses were calculated as "not very likely (4) and "definitely not" (5). For examining likelihood to take PrEP based upon participant characteristics, a stratified analysis using Mantel-Haenszel chi-square test was undertaken in order to adjust for potential confounding (age), Both unadjusted (age) and adjusted odds ratios with $95 \%$ confidence intervals were calculated. McNemar's test for correlated proportions was used to evaluate differences of likely and unlikely survey responses between those who completed both the initial and the follow-up surveys. The $\mathrm{p}$-values were based on two-tailed test results and $\mathrm{p} \leq 0.05$ was used to define statistical significance.

\section{Results}

\section{Study population}

Among 103 eligible persons who were invited to participate, $65(63.1 \%)$ completed the survey. Reasons for not participating in the survey included no response to telephone or email messages (32 participants); declined participation (5 participants); and incarceration (1 participant). Among survey participants, median age was 36 years (range; $20-52$ years); $88 \%$ were white, $6 \%$ Hispanic, and $1.5 \%$ African American; $65 \%$ had at least a college degree or higher and $27 \%$ had more than high school education. The reported number of sexual partners in the past six months ranged from zero to 150 partners (median 3 partners) and 75\% reported condom use during all sexual encounters (Table 1). During the initial survey, 47 participants $(72.3 \%)$ indicated prior knowledge of PrEP and 5 participants (7.7\%) reported having used PrEP in the past.

\section{Likelihood of using PrEP in the Future}

Participants were most likely to use PrEP in the future if they were in a monogamous relationship with an HIV-infected partner (93.8\%) or had casual sexual partners whose HIV status was unknown (89.1\%). Eighty percent (80\%) of participants would be likely to use PrEP if it was provided free of charge, whereas $89.3 \%$ of participants were unlikely to use PrEP if it cost money out-of-pocket, approximately $\$ 500$, each month. Participants were also less likely to use PrEP if there was a more effective vaccine available (89.2\%) and if there were associated side effects (70.8\%; Figure 1).

Younger participants ( $\leq 36$ years) were less likely to take PrEP if it cost money out-of-pocket each month (OR 0.1; 95\% CI 0.0, 1.0), if PrEP was provided free of charge (OR 0.2; 95\% CI 0.1, 1.0) or if there were possible side effects (OR 0.3; 95\% CI 0.1, 1.0). Higher number of partners ( $>5$ partners) in the preceding six months was associated with decreased likelihood of anticipating use of condoms if taking 


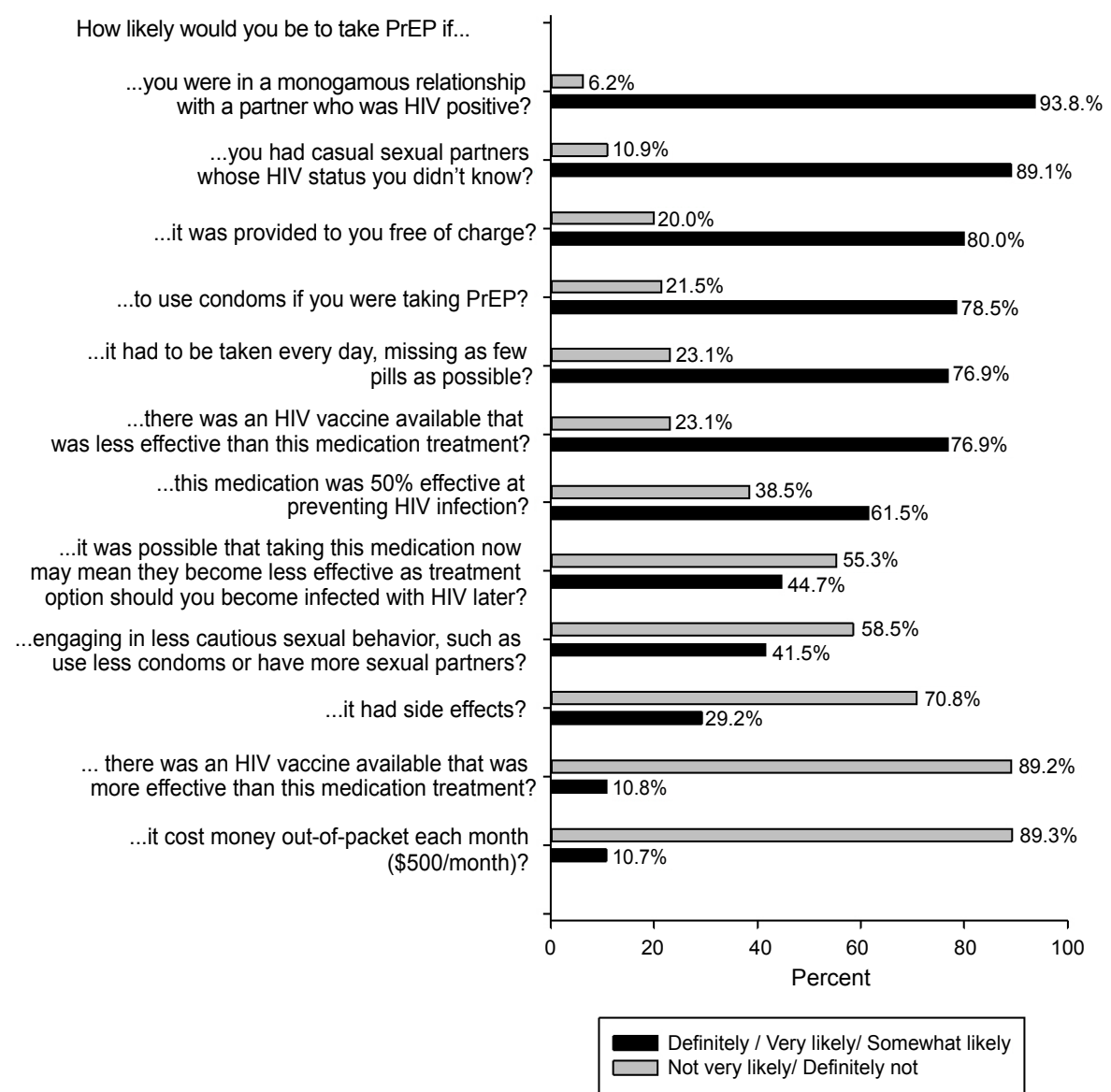

Figure 1: Percentage of Likelihood of PrEP Use, by question $(n=65)$. The percentage of responses in each category or the survey question in Appendix 1 is provided on the $\mathrm{x}$-axis.

PrEP (aOR 0.2; 95\% CI 0.1, 0.8). There were no associations between likelihood to use PrEP and level of education, prior knowledge of PrEP or number of sexual partners (Table 2). Fifty-four participants (83.1\%) completed both the initial and six month follow-up surveys. There were no statistically significant changes in survey responses between the initial and follow-up surveys (data not shown).

\section{Discussion}

The present study sought to uncover perceived barriers to PrEP use among high-risk HIV-seronegative men. We found that prior PrEP use was uncommon and cost, side effect profile, and potential availability of an effective vaccine were found to be associated with decreased willingness to use PrEP in the future. Presumed PrEP use was found to have some influence on anticipated condom use in the future among those with greater than five partners in the past six months. Importantly, younger age was associated with decreased willingness to use PrEP regardless of costs or side effects. This finding is in agreement with a prior study which found that older age was associated with greater PrEP interest [16]. Since starting PrEP at an early age would result in a longer total duration of PrEP usage and greater cumulative exposure, decrease willingness to use PrEP in the younger age group could reflect concerns about potential toxicities over one's lifetime. Additional studies are needed to better understand why younger sexually active men are less willing to consider PrEP usage and evaluate interventions to increase PrEP use in this high HIV-risk group.
Few studies to date have examined multiple social, economic, and other factors influencing the intent to take PrEP among those at risk for HIV infection. Krakower, et al. found that shortly after the iPrEx study results were released awareness of PrEP was limited and usage was uncommon [16]. Fuchs, et al. previously examined interest in PrEP, financial barriers, and the effect of taking PrEP on participation in the HVTN 505 trial. They found that a minority of participants expressed intent to use PrEP, however that interest significantly increased if PrEP was provided free of charge [17]. Other survey studies have investigated awareness of PrEP among high-risk populations, and demonstrated widely varying levels of awareness across various risk groups with similar attitudes towards cost and side effects $[6,7,9,18]$. Similar to these studies, our study confirms a general awareness of PrEP, and concern for cost and side effects among potential PrEP users as well. Our study is unique in its examination of the influence of sexual practices and behaviour's, personal relationships, and the effect of future PrEP usage on the use of other prevention methods such as condoms. Taken together, these findings confirm the relative lack of uptake of PrEP as a prevention strategy among high risk men and point toward realistic targets to increase PrEP usage for effective prevention of HIV transmission.

Responses from our initial survey demonstrated that those at risk of HIV might be more likely to take PrEP under certain circumstances, such as monogamous discordance or unknown status of numerous 


\begin{tabular}{|c|c|c|c|c|}
\hline \multirow[b]{2}{*}{ How likely would you be to take PrEP if... } & \multicolumn{4}{|c|}{ Odds Ratios (95\% confidence intervals) } \\
\hline & Age & Education $\ddagger$ & PrEP knowledge $\ddagger$ & Sexual risk $\ddagger$ \\
\hline (definitely/very likely/somewhat likely) & $\begin{array}{c}1.0 \text { (ref): }>36 \text { years } \\
\text { [median] }\end{array}$ & $\begin{array}{l}1.0 \text { (ref): HS education } \\
\text { or less }\end{array}$ & $\begin{array}{l}1.0 \text { (ref): no prior } \\
\text { knowledge }\end{array}$ & $\begin{array}{c}1.0 \text { (ref): } \leq 5 \text { partners, } \\
\text { last } 6 \text { months }\end{array}$ \\
\hline ...it was provided to you free of charge? & $\begin{array}{c}0.2 \\
(0.1,1.0) \dagger\end{array}$ & $\begin{array}{c}0.7 \\
(0.2,2.6)\end{array}$ & $\begin{array}{c}0.7 \\
(0.2,3.2)\end{array}$ & $\begin{array}{c}3.3 \\
(0.4,27.7)\end{array}$ \\
\hline ...it cost you money out-of-pocket each month? & $\begin{array}{c}0.1 \\
(0.0,1.0) \dagger\end{array}$ & $\begin{array}{c}3.4 \\
(0.4,31.8)\end{array}$ & $\begin{array}{c}0.4 \\
(0.1,2.4)\end{array}$ & $\begin{array}{c}0.4 \\
(0.0,3.7)\end{array}$ \\
\hline ...it had side effects? & $\begin{array}{c}0.3 \\
(0.1,1.0) \dagger\end{array}$ & $\begin{array}{c}1.2 \\
(0.4,3.7)\end{array}$ & $\begin{array}{c}0.5 \\
(0.2,1.7)\end{array}$ & $\begin{array}{c}0.7 \\
(0.2,2.5)\end{array}$ \\
\hline ...it had to be taken every day, missing as few pills as possible? & $\begin{array}{c}0.6 \\
(0.2,2.0)\end{array}$ & $\begin{array}{c}2.6 \\
(0.8,8.5)\end{array}$ & $\begin{array}{c}1.4 \\
(0.4,5.0)\end{array}$ & $\begin{array}{c}2.1 \\
(0.4,10.9)\end{array}$ \\
\hline ...this medication was $50 \%$ effective at preventing HIV infection? & $\begin{array}{c}0.4 \\
(0.1,1.2)\end{array}$ & $\begin{array}{c}0.7 \\
(0.2,2.1)\end{array}$ & $\begin{array}{c}0.4 \\
(0.1,1.2)\end{array}$ & $\begin{array}{c}0.6 \\
(0.2,1.7)\end{array}$ \\
\hline $\begin{array}{l}\text {...there was an HIV vaccine available that was less effective than } \\
\text { this medication treatment? }\end{array}$ & $\begin{array}{c}0.9 \\
(0.3,2.8)\end{array}$ & $\begin{array}{c}1.8 \\
(0.6,6.0)\end{array}$ & $\begin{array}{c}0.9 \\
(0.3,3.4)\end{array}$ & $\begin{array}{c}1.2 \\
(0.3,5.3)\end{array}$ \\
\hline $\begin{array}{l}\text {...there was an HIV vaccine available that was more effective than } \\
\text { this medication treatment? }\end{array}$ & $\begin{array}{c}0.7 \\
(0.1,3.4)\end{array}$ & $\begin{array}{c}1.4 \\
(0.2,7.8)\end{array}$ & $\begin{array}{c}0.5 \\
(0.1,2.3)\end{array}$ & $\begin{array}{c}1.3 \\
(0.2,7.2)\end{array}$ \\
\hline $\begin{array}{l}\text {...you were in a monogamous relationship with a partner who was } \\
\text { HIV positive? }\end{array}$ & $\begin{array}{c}0.3 \\
(0.0,3.2)\end{array}$ & $\begin{array}{c}0.6 \\
(0.1,6.1)\end{array}$ & $\begin{array}{c}1.0 \\
(0.1,10.0)\end{array}$ & - \\
\hline $\begin{array}{l}\text {...you had casual sexual partners whose HIV status you didn't } \\
\text { know? }\end{array}$ & $\begin{array}{c}3.0 \\
(0.5,16.7)\end{array}$ & $\begin{array}{c}0.3 \\
(0.0,2.5)\end{array}$ & $\begin{array}{c}0.4 \\
(0.0,3.5)\end{array}$ & $\begin{array}{c}0.4 \\
(0.1,1.9)\end{array}$ \\
\hline $\begin{array}{l}\text {...it is possible that taking this medication now may mean they } \\
\text { become less effective as a treatment option should you become } \\
\text { infected with HIV in the future? }\end{array}$ & $\begin{array}{c}0.7 \\
(0.2,1.7)\end{array}$ & $\begin{array}{c}0.8 \\
(0.3,2.1)\end{array}$ & $\begin{array}{c}0.7 \\
(0.3,2.2)\end{array}$ & $\begin{array}{c}0.5 \\
(0.1,1.6)\end{array}$ \\
\hline ...to use condoms if you were taking PrEP? & $\begin{array}{c}1.5 \\
(0.5,5.0)\end{array}$ & $\begin{array}{c}0.7 \\
(0.2,2.5)\end{array}$ & $\begin{array}{c}1.1 \\
(0.3,3.9)\end{array}$ & $\begin{array}{c}0.2 \\
(0.1,0.8) \dagger\end{array}$ \\
\hline $\begin{array}{l}\text {...to engage in less cautious sexual behavior, such as use } \\
\text { condoms less or have more sexual partners? }\end{array}$ & $\begin{array}{c}1.4 \\
(0.5,3.7)\end{array}$ & $\begin{array}{c}1.6 \\
(0.6,4.5)\end{array}$ & $\begin{array}{c}0.6 \\
(0.1,2.7)\end{array}$ & $\begin{array}{c}1.4 \\
(0.4,4.7)\end{array}$ \\
\hline $\begin{array}{c}\dagger p \leq 0.05 \\
\ddagger \text { Adjusted for age }\end{array}$ & & & & \\
\hline
\end{tabular}

Table 2: Analysis of participant characteristics and the likelihood to take PrEP.

partners. This may indicate an intention to take PrEP on an "as-needed" or anticipated basis, rather than daily as continuous prophylaxis. The efficacy of intermittent PrEP usage is unknown but is under investigation in ongoing studies [19]. In a sub-study of the iPrex trial, a minimum intracellular concentration of the active form of tenofovir was found to be associated with a 90\% reduction in HIV acquisition as compared to placebo [20]. The STRAND trial examined the effect of directly observed various dosing, including two days a week, four days a week, and seven days a week, and observed that varying intracellular drug concentrations corresponded to an HIV risk reduction of $76 \%$ for two doses a week, $96 \%$ for four doses per week, and $99 \%$ for seven doses per week [18]. These studies demonstrate that intracellular drug concentrations, as determined by dosing frequency, are correlated with decreased acquisition of HIV while taking PrEP in controlled environments. If "as-needed" dosing becomes the intention of those targeted for continuous PrEP use, it may be hypothesized that the protective effect is less than previously seen in controlled studies and thus less predictable, and further studies would be needed to evaluate the as-needed basis of prophylaxis. Lastly, as with the treatment of HIV, intermittent dosing brings a greater chance of developing antiretroviral drug resistance. Successful prevention of HIV continues to demand a combination of behaviour change and medical intervention; without this two-pronged approach, an as-needed prophylactic medication may bring greater risk than benefit.

Limitations of the present study include a relatively small sample size and a single site design, which potentially limits generalizability to other high-risk populations. Other limitations include bias by the interviewers in conducting phone surveys with preferential intonation or further explanations of questions. An in-person written survey could have avoided this potential interviewer bias. The survey question concerning prior PrEP use could have been interpreted as also including post-exposure prophylaxis, and thus our finding of $7.7 \%$ prior PrEP usage might be an over estimate.. The survey question concerning outof-pocket expenses assumed minimal coverage by third party providers (out-of-pocket costs of $\$ 500$ per month) and we did not assess the potential impact of lesser out-of-pocket costs. Participants for the HVTN 505 trial, from which our participants were recruited, were selfselected through advertising, and therefore a selection bias of those who are inclined to seek out opportunities for prevention, research, and new resources is present for our study population as well.

PrEP has been shown in several large-scale studies to reduce the transmission of HIV by $44-75 \%$ and has gained FDA approval for use in the US and recent CDC clinical practice guideline recommendation [21]. However it has yet to gain acceptance as a common prevention method in at-risk communities. While previous studies have found the interest in PrEP to be high, [9] the reality of instituting PrEP on a large scale poses more challenges than patient interest alone. The barriers to PrEP identified by the present study speak to a relative lack of readiness among high-risk populations to widely accept a daily preventative medication regimen. As discussed above, the inadequate intracellular drug levels attained by intermittent "as-needed" dosing would likely not confer full protection, as several studies have also demonstrated $[1,18]$. The next step in HIV chemo-prevention is the development of depot formulations of prophylactic medication. Animal models have shown early success at preventing vaginal transmission of simian HIV (SHIV) when macaques were given monthly injections of a long-acting formulation of the HIV integrase inhibitor GSK744 [22]. Additionally, early development of rilpivirine, a non-nucleoside reverse transcriptase inhibitor, as a depot formulation has been considered [23]. While high cost and side effect profiles may still accompany depot formulations at least initially, a long-acting injection may be more palatable to those at increased risk of HIV and more widely accepted as a population- 
Citation: Gersh JK, Fiorillo SP, Burghardt L, Nichol AC, Thrun M, et al. (2014) Attitudes and Barriers towards Pre-Exposure Prophylaxis (Prep) among High-Risk HIV-Seronegative Men who have Sex with Men. J AIDS Clin Res 5: 335. doi:10.4172/2155-6113.1000335

based form of prevention. Regardless of how PrEP is delivered, if PrEP is to have major impact on transmission of HIV-1, expanded efforts to decrease cost and increase community awareness of PrEP safety and efficacy are needed.

\section{Acknowledgment}

The authors would like to thank Denver Public Health for their generous financial support towards incentives for our participants upon completion of the surveys. Additionally, this study would not have been possible without the assistance of Ms. Graham Ray, Ms. Christine Griesmer, and Ms. Cathi Basler in recruiting participants for the survey.

\section{References}

1. Grant RM, Lama JR, Anderson PL, McMahan V, Liu AY, et al. (2010) Preexposure chemoprophylaxis for HIV prevention in men who have sex with men. N Engl J Med 363: 2587-2599.

2. Baeten JM, Donnell D, Ndase P, Mugo NR, Campbell JD, et al. (2012) Antiretroviral prophylaxis for HIV prevention in heterosexual men and women. N Engl J Med 367: 399-410.

3. Thigpen MC, Kebaabetswe PM, Paxton LA, Smith DK, Rose CE, et al. (2012) Antiretroviral preexposure prophylaxis for heterosexual HIV transmission in Botswana. N Engl J Med 367: 423-434.

4. Van Damme L, Corneli A, Ahmed K, Agot K, Lombaard J, et al. (2012) Preexposure prophylaxis for HIV infection among African women. $\mathrm{N}$ Engl $\mathrm{J}$ Med 367: 411-422.

5. Okwundu $\mathrm{Cl}$, Uthman OA, Okoromah CAN (2012) Antiretroviral pre-exposure prophylaxis (PrEP) for preventing HIV in high-risk individuals. Cochrane Database Syst Rev 7: CD007189.

6. Mustanski B, Johnson AK, Garofalo R, Ryan D, Birkett M (2013) Perceived likelihood of using HIV pre-exposure prophylaxis medications among young men who have sex with men. AIDS Behav 17: 2173-2179.

7. Barash EA, Golden M (2010) Awareness and use of HIV pre-exposure prophylaxis among attendees of a seattle gay pride event and sexually transmitted disease clinic. AIDS Patient Care STDS 24: 689-691.

8. Aghaizu A, Mercey D, Copas A, Johnson AM, Hart G, et al. (2013) Who would use PrEP? Factors associated with intention to use among MSM in London: a community survey. Sex Transm Infect 89: 207-211.

9. Mimiaga MJ, Case P, Johnson CV, Safren SA, Mayer KH (2009) Preexposure antiretroviral prophylaxis attitudes in high-risk Boston area men who report having sex with men: limited knowledge and experience but potential for increased utilization after education. J Acquir Immune Defic Syndr 50: 77-83.

10. Al-Tayyib AA, Thrun MW, Haukoos JS, Walls NE (2014) Knowledge of preexposure prophylaxis (PrEP) for HIV prevention among men who have sex with men in Denver, Colorado. AIDS Behav 18 Suppl 3: 340-347.
11. National Institute of Health (2013) NIH Discontinues Immunizations in HIV Vaccine Study.

12. Hammer SM, Sobieszczyk ME, Janes H, Karuna ST, Mulligan MJ, et al. (2013) Efficacy trial of a DNA/rAd5 HIV-1 preventive vaccine. N Engl J Med 369: 2083 2092.

13. HIVIAIDS: Basic Statistics.

14. MMWR (2001) HIV and AIDS - 1981-2000.

15. Harris PA, Taylor R, Thielke R, Payne J, Gonzalez N, et al. (2009) Research electronic data capture (REDCap)--a metadata-driven methodology and workflow process for providing translational research informatics support. J Biomed Inform 42: 377-381.

16. Krakower DS, Mimiaga MJ, Rosenberger JG, Novak DS, Mitty JA, et al. (2012) Limited Awareness and Low Immediate Uptake of Pre-Exposure Prophylaxis among Men Who Have Sex with Men Using an Internet Social Networking Site. PLoS ONE 7(3): e33119.

17. Fuchs JD, Sobieszczyk ME, Madenwald T, Grove D, Karuna ST, et al. (2013) Intentions to use preexposure prophylaxis among current phase $2 \mathrm{~B}$ preventive HIV-1 vaccine efficacy trial participants. J Acquir Immune Defic Syndr 63: 259262

18. Liu AY, Kittredge PV, Vittinghoff E, Raymond HF, Ahrens K, et al. (2008) Limited knowledge and use of HIV post- and pre-exposure prophylaxis among gay and bisexual men. J Acquir Immune Defic Syndr 47: 241-247.

19. Amico KR (2012) Adherence to preexposure chemoprophylaxis: the behavioral bridge from efficacy to effectiveness. Curr Opin HIV AIDS 7: 542-548.

20. Anderson PL, Glidden DV, Liu A, Buchbinder S, Lama JR, et al. (2012) Emtricitabine-tenofovir concentrations and pre-exposure prophylaxis efficacy in men who have sex with men. Sci Transl Med 4: 151ra125.

21. Centers for Disease Control and Prevention (2014) Pre-exposure prophylaxis for the prevention of HIV infection in the United States - 2014: A clinical practice guideline.

22. Garcia-Lerma JG (2014) Monthly GSK744 Long-Acting Injections Protect Macaques Against Repeated Vaginal SHIV Exposures. 21st Conference on Retroviruses and Opportunistic Infections (CROI). Boston, March 3-6. Abstract 40LB.

23. Johnson \& Johnson (2014) Janssen R\&D Ireland Announces Agreement with PATH for Early Development of Rilpivirine in Long-Acting Formulation for Potential Prophylactic Intervention. 